Revue de l'Institut des langues et cultures

d'Europe, Amérique, Afrique, Asie et Australie

28 | 2017

Passages, ancrage dans la littérature de voyage

\title{
De l'Inde à la Provence : la poésie, ultime ancrage de Lawrence Durrell
}

From India to Provence: poetry as Lawrence Durrell's last abode

\author{
Isabelle Keller-Privat
}

\section{OpenEdition}

Journals

Édition électronique

URL : http://journals.openedition.org/ilcea/4153

DOI : 10.4000/ilcea.4153

ISSN : 2101-0609

\section{Éditeur}

UGA Éditions/Université Grenoble Alpes

\section{Édition imprimée}

ISBN : 978-2-84310-374-2

ISSN : 1639-6073

Référence électronique

Isabelle Keller-Privat, « De I'Inde à la Provence : la poésie, ultime ancrage de Lawrence Durrell », ILCEA [En ligne], 28 | 2017, mis en ligne le 06 mars 2017, consulté le 21 avril 2019. URL : http:// journals.openedition.org/ilcea/4153; DOI : 10.4000/ilcea.4153

Ce document a été généré automatiquement le 21 avril 2019

(C) ILCEA 


\title{
De l'Inde à la Provence : la poésie, ultime ancrage de Lawrence Durrell
}

\author{
From India to Provence: poetry as Lawrence Durrell's last abode
}

\author{
Isabelle Keller-Privat
}

1 Lawrence Durrell, né en Inde, à Jullundur ${ }^{1}$, en 1912 de parents anglo-irlandais, consacra son premier ouvrage de jeunesse, Pied Piper of Lovers, au récit d'une enfance indienne marquée par un douloureux exil en Angleterre : celle de Walsh Clifton, son alter ego. Or, au fil des publications l'ancrage indien se fait moins explicite, les premiers romans étant interdits de réédition par l'auteur, comme si celui-ci cherchait à cacher non pas tant son histoire que l'histoire de cette histoire, une fiction sans doute jugée trop transparente. Et pourtant l'Inde ne cesse de faire retour, tant dans la fiction que dans les nombreuses interviews que l'auteur donne : elle est l'alpha et l'oméga de l'écriture, un point d'ancrage qui est aussi un point de départ. En retraçant le cheminement de l'écriture durrellienne à partir du tout premier récit, Pied Piper of Lovers, en comparant l'écriture fictionnelle et celle de l'essai critique "From the Elephant's Back », en suivant les réécritures de l'Inde natale jusqu'au dernier opus, Caesar's Vast Ghost, son hommage à la Provence, nous essaierons de comprendre comment s'accomplit le passage d'une perspective autobiographique sur son ancrage indien à une perspective symbolique et littéraire. Quels sont les enjeux esthétiques et stylistiques d'une écriture qui tente dans un premier temps de dire la douleur du déracinement avant de la déplacer et de la métamorphoser pour en faire son matériau privilégié ? Nous verrons comment le premier roman de Lawrence Durrell nous révèle la nostalgie d'une enfance indienne pour la masquer aussitôt. Ainsi le discours critique que déploie son article «From the Elephant's Back » passe par le détour de la biographie pour exposer une réflexion littéraire qui ne saurait ignorer l'enfance indienne mais qui la retravaille pour en faire cette voie d'accès symbolique à la littérature. L'ancrage indien de Lawrence Durrell devient alors le lieu d'une méditation littéraire et philosophique qui transforme le temps et l'espace en objets symboliques à partir desquels l'écrivain rejoint, dans l'œuvre poétique de son dernier opus, son moi intime. 
2 Le foyer indien du jeune écrivain est à la fois le lieu de projection d'un ancrage désiré et d'un arrachement douloureux qui transparaît dès sa première œuvre, Pied Piper of Lovers, où le jeune héros médite sur le rapatriement de tous ceux qu'il s'apprête à suivre :

He knew that England was where everyone should go; that was the one dream that induced the colonial to save his pay [...] He remembered the Abbis family: they had left for England last summer. He remembered their exultation and happiness. Mr Abbis was huge and broadshouldered and as brown as wood-smoke; he had chattered for weeks on end of going home; of getting his daughters educated at home; of settling down at home; always "home." [...] Walsh found it a peculiarly inspiring word, but applied to England it meant less than nothing. (Durrell, 2008 [1935,] : 55)

Outre la teneur biographique que le lecteur devine aisément derrière le personnage de ce jeune garçon qui tente de se représenter l'Angleterre, on ne peut ignorer le soin apporté à l'écriture qui laisse transparaître les préoccupations littéraires du jeune écrivain, ni les questions éditoriales qui font de ce texte le lieu ambivalent d'un ancrage et d'un passage annonçant les grandes lignes de l'œuvre à venir.

4 De nombreux critiques ont déjà analysé les fortes similitudes entre le destin de Walsh et celui du jeune Durrell, interprétant le personnage comme un «protagoniste autobiographique » (Gifford, 2008: x) que l'auteur aurait par la suite cherché à oublier en refusant que le roman soit réédité. On ne saurait cependant manquer d'être également sensible à l'écriture qui se construit: le morcellement des phrases marqué par l'emploi des points virgules ou des deux points, la répétition du syntagme " he remembered » ainsi que d'unités lexicales choisies (England, Abbis et home) impriment un rythme qui deviendra caractéristique de la prose durrellienne et laissent deviner l'humour à la fois tendre et tragique qui imprègne toute l'œuvre. L'anamnèse est aux fondements d'un ancrage temporel, spatial et affectif qui doue le personnage d'authenticité, participant de la création d'un être qui, par la force des mots, traverse le temps et l'espace. Se remémorant l'attente joyeuse de la famille partie, Walsh ramène dans le temps de la narration un personnage déjà disparu dont la silhouette massive et le teint sombre incarnent une Inde qui s'effondre. L'ancrage présent du jeune homme devient ainsi précaire, comme miné par le départ des voisins. L'ancrage à venir est également compromis dans cette description pleine d'humour de M. Abbis qui sera dès son arrivée, on le devine, considéré comme un étranger dans son propre pays. Le passage qui s'ouvre sur «England» et se referme sur le mot «home» semble miné en son centre par l'apparition monumentale de «Abbis », suggérant l'abîme de nostalgie et de solitude qui creusera l'Angleterre de Lawrence Durrell.

5 La longue description des dernières images de la traversée qui ouvre le premier chapitre $\mathrm{du}$ deuxième livre de Pied Piper of Lovers explore le douloureux arrachement du personnage qui, malgré ses efforts, ne parvient pas à se réjouir de son nouveau port d'attache :

[...] he was not impressed [...] he told himself bitterly that it was smaller than he had imagined! [...] The others were happy [...] peering out upon the promised land [...] experiencing that emotion of country-love which is occasioned in exiles by the sight of the Dover cliffs. (Durrell, 2008 [1935] : 109)

6 La découverte de l'Angleterre suscite chez le jeune garçon non pas un sentiment d'adhésion mais une aliénation profonde qui se marque par un retournement de la dichotomie "us/them » codifiant traditionnellement la société coloniale. Les " autres » deviennent ainsi tous ceux qui, se réjouissant de rentrer au pays, incarnent l'autorité de 
l'Empire dont Walsh se dissocie en tentant d'établir sur le pont du navire un contact avec l'ayah qui découvre en même temps que lui les côtes anglaises :

A small, gentle-looking ayah came and stood beside the boy, and peered out across the water [...]

He felt a little sick as he noticed her flat Mongol features, sick with an undefined regret, as though the beauty of the hills which he had left behind for ever still worked in him. [...]

"You are of the hills?"

"Yes. Of Nepal. In the service of mem-sahib."

[...]

He said shyly:

"I, too, am from the hills. Kurseong."

[...] He would have liked to talk to her about the places that already seemed remote, but she seemed to regard him as yet another of the alien race with whom she had nothing in common save the coincidence of a common dwelling; a birth-place and a country for her, for him no more than a temporary house. (Durrell, 2008 [1935] : 111)

7 Dernier contact tangible avec le pays qu'il vient de quitter, l'ayah silencieuse signe la perte de la terre nourricière qui l'a accueilli et la double aliénation du personnage qu'analyse James Gifford :

This perspective places Walsh in the doubly estranged position of exile from India and life in an England that would not accept him. It is also telling that this young ayah's recognition of race leads immediately to thoughts of nationality, a fact that could not have been overlooked by the young Durrell, who was sent to England in the same year that Nepal's independence was recognized by Britain. (Gifford, 2008 : XIV)

L'histoire intime et la grande Histoire vont progressivement lui refuser l'Inde ${ }^{2}$, le contraignant à faire doublement la cruelle expérience de la perte de ce foyer, de ce « home ", auquel se substitue une infinité de «temporary house[s]» où il sera toujours « en exil d'exil », comme l'explique Corinne Alexandre-Garner (2003:56).

9 L'histoire de la publication de Pied Piper of Lovers montre l'ambiguïté de cet exil, à la fois subi, réitéré et consenti. Ce texte de jeunesse auquel Durrell apportera ses dernières corrections depuis l'île de Corfou, alors qu'il a renoncé à faire de l'Angleterre son foyer malgré les injonctions paternelles et qu'il construit simultanément une carrière de romancier et de poète ${ }^{3}$, sera en effet interdit de réédition, tout comme le suivant, Panic Spring, jusqu'à la mort de l'auteur ${ }^{4}$. Lawrence Durrell repousse ainsi aux confins de son œuvre l'un de ses très rares textes autobiographiques, interdisant tout accès aux origines de son ancrage indien, répétant la perte dans laquelle s'enracine l'écriture. Pied Piper of Lovers se donne ainsi en quelque sorte comme l'envers du texte d'E. M. Forster : non point A Passage to India mais A Passage to England, le texte révèle l'hypocrisie et l'absurdité d'un monde qui disparaît, le paradis perdu de l'enfance et les espoirs déçus, ne laissant derrière lui que les échos lointains et troublants d'un ancrage sensuel, affectif et éthique qui hante toute l'œuvre durrellienne.

Alors qu'il réside en France depuis déjà vingt-cinq ans, installé en Provence où il a publié récits de voyages et recueils de poèmes inspirés de sa vie en Grèce, ainsi que le célèbre Quatuor d'Alexandrie et les deux premiers volumes du Quintette d'Avignon, Lawrence Durrell rédige pour la première fois en français une conférence prononcée en 1981 à Paris au Centre Pompidou qui sera par la suite publiée en anglais ${ }^{5}$. Dans ce texte, qui relève à la fois de la lettre et de l'essai, Durrell se présente comme cet « Anglo-indien » élevé dans l'ombre de Kipling :

[...] my thinking is coloured by the fact that I am a colonial, an Anglo-Indian, born into that strange world of which the only great poem is the novel Kim by Kipling. I was brought up in 
its shadow, and like its author I was sent to England to be educated. (Durrell, 2015 [1981] :

1-2)

11 Lovers, voici un discours autobiographique pleinement assumé : «I would prefer to present my case in terms of biography " (Durrell, 2015 [1981] : 1) précise l'auteur au tout début de son discours. Voici donc réécrit dans un discours autobiographique A Passage to England dans lequel transparaît une nouvelle fois l'inversion des codes de l'Empire : l'enfant de sahib se décrit comme "coloured», adoptant la posture de l'étranger, comme si l'Inde avait laissé son empreinte dans sa chair et dans son âme. À l'instar du jeune Kim, passeur révélant tout au long de son parcours picaresque les richesses de l'Inde multiculturelle aux lecteurs ignorants de l'Empire, Durrell tente, comme il l'explique tout au long de cette conférence, de joindre l'Orient et l'Occident. Son don pour les langues qui lui permet de naviguer entre l'hindi et l'anglais d'abord, puis entre l'anglais, le français et le grec, prépare le lecteur à un autre passage qui dédouble celui du voyageur : «My first language was Hindi» (Durrell, 2015 [1981] : 2) annonce le début de la conférence, avant de nous conduire à l'affirmation centrale : «[...] literature [...] became my passport » (Durrell, 2015 [1981] : 6). Entre ces deux moments du texte s'opère le subtil déplacement par lequel l'écrivain quitte le simple récit biographique pour s'essayer à une écriture qui est proprement nouvelle pour lui et par laquelle il devient son propre objet de méditation. Déployant un regard rétrospectif, il révèle indirectement au lecteur son ambition d'écrivain puisée aux sources mêmes du monde indien qui marqua son enfance :

Obviously, to live in a country where the whole population, both civil and ecclesiastical was trying implacably to seek a fulcrum of repose at the heart of reality, a country where people were living alongside nature and not in tangence to it, gives off a very powerful flavour, permeating the air. (Durrell, 2015 [1981] : 4).

C'est en se laissant toucher par la quête de ce " pivot de la sérénité au cœur de la réalité » qui semble imprégner l'air qu'il respire que l'écrivain s'ancre dans la nature du monde qu'il habite et définit sa propre quête. Cette inspiration indienne le conduit à interroger tant les grandes découvertes scientifiques de son temps que les subtilités de la pensée orientale et grecque à travers lesquelles il s'efforce de déceler les grands invariants de la philosophie antique. L'Inde durrellienne émerge ainsi comme ce texte à déchiffrer, aux carrefours des influences orientales et classiques, lieu de déposition d'un savoir véritable qui échappe au langage. En se détournant d'un propos qui s'annonçait dès le début de la conférence comme biographique, Lawrence Durrell revient donc par des chemins détournés vers le centre de ses intérêts : «the theory and the practice of fiction " (Durrell, 2015 [1981] : 1). Ainsi s'élabore une écriture caractéristique de l'essai, attentive, explique Marielle Macé «à la processualité de la pensée et aux formes dans lesquelles celle-ci se dépose » (2006: 36) et par laquelle l'écrivain se ré-écrit. "From the Elephant's Back» révèle donc une Inde qui constitue en réalité un creuset affectif et philosophique que l'écrivain a toujours porté en lui. "One part of me has remained a child of the jungle " (Durrell, 2015 [1981] : 2), affirme-t-il, nous préparant au récit de son déracinement : « It was of course a dramatic wrench to leave India [...] » (Durrell, 2015 [1981] : 6), « [...] it was a great wrench leaving Sadu »(Durrell, 2015 [1981]: 6) répète-t-il à deux paragraphes d'intervalle pour clore le récit de ses excursions enfantines sur le dos de l'éléphant Sadu. On comprend en lisant ce double épilogue qui fait écho au titre de la conférence que l'Inde n'est plus tant un ancrage biographique que symbolique dont l'écrivain fait son deuil grâce à l'écriture. L'image du petit garçon soulevé dans les airs par la trompe de l'éléphant et voyageant au rythme de son pas flottant fonctionne alors comme un

ILCEA, 28 | 2017 
emblème poétique: "But the proverb says that whoever sees the world from the back of an elephant learns the secrets of the jungle and becomes a seer. I had to be content to become a poet, but it was enough for one life. » (Durrell, 2015 [1981] : 4).

La biographie sert alors moins à dessiner les origines du sujet qu'à révéler le destin de l'écrivain: Lawrence Durrell est ce poète-voyant dont l'écriture se nourrira de la poésie symboliste française comme de la poésie grecque antique mais dont le sort était en quelque sorte déjà écrit avant même que ne commence son apprentissage, loin des premiers contreforts de l'Himalaya. C'est depuis le dos du jeune éléphant orphelin que Lawrence Durrell construit son propre mythe à travers lequel le biographique se trouve transfiguré par le symbolique. Se dessine alors la figure du poète à naître qui saura conjuguer ce qu'il nomme "the two types of consciousness [...] an ambivalence of vision" (Durrell, 2015 [1981] : 2) qui nourrissent déjà ses rêves d'enfant : « [...] I wanted a typewriter and if such a thing had been possible, an elephant » (Durrell, 2015 [1981] : 7). Ce Noël-là son père lui offrit sa première machine à écrire et les œuvres complètes de Shakespeare qui le porteront vers le monde de la littérature, lui permettant d'accomplir le passage de l'Inde vers le monde occidental. Pourtant, il n'aura de cesse de retrouver l'Inde: «[...] I was really trying to find my way back to India [...] I was half-way home » (Durrell, 2015 [1981] : 8-9), explique-t-il en décrivant son enfance anglaise et sa découverte de la philosophie grecque.

Lawrence Durrell exposant son enfance indienne pour mieux approfondir sa théorie sur l'art de la fiction prépare ainsi son lecteur-auditeur à la forme hybride de l'essai, à savoir un discours qui oscille entre l'affirmation du savoir et l'exploration du « je » de l'écrivain, qui se construit fondamentalement comme ce long voyage au cours duquel se cristallisent les infimes parcelles de lui-même que l'auteur tente de remembrer : petit garçon juché sur le dos d'un éléphant, jeune écolier, apprenti écrivain sont autant d'empreintes qui le ramènent à la fois vers sa terre natale et vers celle de l'écriture, le Languedoc : «The track ran through landscapes of dreams, dit-il du petit train à vapeur de Darjeeling, just as the abandoned railway lines of the Gard, near where I live in the South of France, along which I walk and reflect also » (Durrell, 2015 [1981] : 5). En suivant la voie de chemin de fer, tout comme il suit les routes nationales dans l'incipit de l'essai, en se retournant vers le petit train, ce "Toy train » construit par les ingénieurs britanniques à flanc de coteaux, l'écrivain ne se saisit pas seulement d'une image passée : il cisèle un motif à partir duquel, comme le rappelle très justement James Gifford, s'élabore l'incipit de Monsieur et de Quinx. Le premier et le dernier volume du Quintette d'Avignon mettent en effet en scène des narrateurs qui observent le monde depuis la fenêtre du train dont le rythme obsédant préside à la fois à la recomposition de la mémoire et à la décomposition du texte. Le discours laisse ainsi présager d'une écriture qui joue avec les limites, mêlant réflexion analytique, images poétiques et ébauches de motifs romanesques. La voix de l'auteur affirme son refus de tout assujettissement à une forme achevée : ni discours savant, ni analyse didactique, ni étude critique, le texte se lit en marge des œuvres déjà publiées et à venir, comme ce « texte provisoire ou supplémentaire » dont parle Marielle Macé (2006: 48) c'est-à-dire, un texte des limbes, à l'image du chemin de fer abandonné, et qui annonce l'écriture de l'essai. Loin d'offrir un tableau achevé de l'œuvre de l'auteur et de son parcours, ce texte est à l'image des chiffons tachés par les brosses du peintre qui donnent couleur et lumière à des fragments d'être : «the paint rags upon which the artist wipes his brushes once the painting is complete » (Durrell, 2015 [1981] : 16). 
comprend alors, en reconnaissant de nombreuses références à l'Inde dans l'hommage que Durrell commença à écrire quelques années plus tard sur la Provence, que ce dernier renoue ici simultanément avec l'ancrage oriental à jamais perdu et avec une forme bien particulière d'écriture, celle de l'essai ainsi définie par Marielle Macé :

Dramatisation de la limite, exploitation de l'espace marginal, digressions, manifestation de l'inachèvement, figures de réflexivité, de non-clôture... le texte matérialise son histoire, manifeste sa précarité, thématise son incertitude. [...] En cela un essai vient toujours en lieu et place d'autre chose [...] Il offre un délai [...].

(2006: 48)

De façon significative, la Provence est décrite dès le deuxième chapitre de Caesar's Vast Ghost comme l'espace d'une initiation poétique et métaphysique dont les caractéristiques ("self-abnegation», "ascetism», Durrell, 1990: 21) héritées du monde grec («Greek frugality and sparseness », Durrell, 1990 : 63) font écho à l'abstraction et à l'épurement de la pensée indienne exposée au premier chapitre :

[...] those who recognize that the primal condition in nature is one of impermanence. They practice anchoring their minds in its flux as the basis for a coherent philosophic life. The moral posture in the matter of death is most important. (Durrell, $1990: 14$ )

Or, toute l'œuvre est habitée par la question du rapport à l'impermanence des choses et à la mort qui détermine, selon Lawrence Durrell, notre rapport au bonheur : qu'il s'agisse du narrateur de Caesar's Vast Ghost affirmant « one feels that real bliss, the smiling silence of pure transcendance, is Asiatic » (Durrell, $1990: 14$ ) ou du châtelain fictif Aldo déclarant « [...] I wish to announce the possibility of tremendous happiness-total bliss [...] (Durrell, 1990 : 37), les différentes voix désignent un Orient au cœur du texte, le lieu d'un avènement vers lequel l'écriture se tourne et nous retourne. Il ne s'agit alors plus de déchiffrer la présence d'un rapport nostalgique à l'Inde mais plutôt l'héritage esthétique et philosophique qui marque le texte de son empreinte. Le projet d'écriture de Caesar's Vast Ghost se lit comme un hommage à cette philosophie paradoxale de la fluidité du monde qui révèle aux âmes patientes le secret d'une forme d'éternité :

If Jérôme had fixed one point from which to judge the whole subject of Provence (which, despite the abandoned book, is ever-present, always on the boil), it is the oft-invoked pérennite des choses, the feeling that all history is endlessly repeating itself, perpetuating itself, not in the form of a chronological ribbon, a linear form, but in a momentous simultaneousness. (Durrell, 1990 : 34-5)

De façon symptomatique, l'essence de la Provence est formulée à travers le regard de Jérôme, le vagabond, berger, poète, historien, figure fictive et passagère. L'écriture de cet extrait, qui insère entre parenthèses un aparté du narrateur dont les choix lexicaux créent un écho inattendu avec la focalisation interne de Jérôme (ever-present//oft-invoked ), contribue à façonner cet univers mouvant au sein duquel les espaces temporels mais aussi linguistiques se répondent (l'anglais se trouve ainsi repris par le français), instaurant une relation mimétique avec son objet. «La simultanéité capitale » à laquelle aboutit la phrase répond au "point fixé " par Jérôme, comme si la phrase, à l'instar de l'essence de la Provence, se repliait sur elle-même. On comprend ainsi la tâche impossible que s'est fixé « le livre abandonné » et la relation paradoxale qui se crée avec le livre réel. Car si Caesar's Vast Ghost constitue bien l'aboutissement du projet de l'écrivain il n'en est pas moins un échec témoignant de l'impossible rêve. À la "pérennité des choses " qui constitue l'âme de la Provence, l'écriture répond par un récit brisé, entrecoupé de citations extraites des ouvrages de la Provence consultés par l'auteur, mêlant des bribes de fiction jamais développées, construisant un texte qui témoigne effectivement du 
retour de l'écriture sur elle-même, comme si celle-ci, contemplant dans le miroir de la bibliothèque ses propres sources, devenait incapable d'organiser une forme linéaire et cohérente, un récit doué d'une essence. Au texte abandonné succèdent donc les fragments de textes qui se sont déposés dans la mémoire de l'écrivain. Qu'il s'agisse de textes d'écrivains comme Stendhal, Mistral ou Sade, de textes d'historiens ou de textes classiques, des descriptions architecturales, des récits de fondation de cités antiques ou du rituel de la tauromachie, Caesar's Vast Ghost ne nous dévoile pas tant la Provence que la tentative de l'auteur de s'inscrire dans la reproduction des textes antérieurs, dans la répétition de scènes dont le récit est devenu un topos de la Provence. Il semble que l'auteur renonce à la forme du récit, se contentant de ce qu'il appelle un "patchwork» (Durrell, 1990 : 149, 183) à l'image du pays qu'il décrit et dont les frontières mouvantes au fil des siècles ne sont jamais clairement définies par le récit durrellien. La carte cachée se trouve en réalité à la fin du premier volume du livre de Theodore Cook, Old Provence, publié en 1905 , et qui faisait partie de la bibliothèque de l'auteur ${ }^{6}$. On trouve en fin d'ouvrage deux cartes : la première schématise un delta du Rhône élargi allant de Foix à Monaco et culminant à Valence et qui correspond à la provincia romaine. La deuxième, plus détaillée indique les sites d'intérêt commentés dans l'ouvrage et que Durrell reprendra en grande partie.

On comprend alors que Lawrence Durrell, dans cette dernière œuvre, abandonne non seulement le récit mais également sa position de narrateur au sens où la définit Umberto Eco, c'est-à-dire "le rôle d'une sorte de démiurge qui crée un monde » (2013: 20). Ce monde est en effet moins créé que dépecé à travers un travail citationnel qui participe d'une création désarticulée, mêlant écriture historique, gothique et carnavalesque pour mieux déjouer les attentes du lecteur (Keller-Privat, 2015). La voix de l'auteur semble parasitée tant par les voix authentiques que par celles des personnages fictifs en proie au délire ${ }^{7}$. Le texte se délite pour offrir une succession de fragments arrachés au réel, comme autant de tessons imparfaitement juxtaposés constituant ce que Jean-Christophe Bailly, expliquant Le Livre des passages de W. Benjamin, appelle "une sorte de déposition des choses en elles-mêmes» qui obéit à une "technique de la proximité » (2015: 23). La proximité des anecdotes historiques ou fictives, réalistes ou grotesques qui composent Caesar's Vast Ghost n'est alors pas sans évoquer la technique de l'archéologie: en rapprochant des morceaux textuels épars, en conjoignant des épisodes temporels discontinus, l'œuvre recompose moins un paysage, moins une réalité historique, que l'écho des traces que ses multiples écritures ont laissées dans la conscience de l'auteur. On songe à l'archéologie du collectionneur décrit par W. Benjamin, qui « fait son affaire de l'idéalisation des objets [...] [et] se plaît à susciter un monde non seulement lointain et défunt mais en même temps meilleur [...]»(1939: 11). Lawrence Durrell se fait collectionneur de textes, d'objets, d'images qu'il juxtapose pour tenter de dire une Provence empreinte de nostalgie, un monde disparu dont la mort est le sceau de la beauté :

I think even the modern tourist feels the deep introspective undertow of a certain melancholy, which prompts one to ask an unusual question which sounds more medieval than Roman, namely, 'Is there not something profoundly morbid at the heart of all beauty?' (Durrell, 1990 : 143) idéalisé - non cette fois celui de l'enfance de l'auteur mais celui de l'enfance du monde devient le lieu où Lawrence Durrell se retire une dernière fois et où "dérivent les fantasmagories de l'intérieur [...] [où] il assemble les régions lointaines et les souvenirs du 
passé » (Benjamin, 1939: 11). Si le récit se trouve évidé c'est parce qu'il est creusé de l'intérieur par ces objets visuels et textuels qui ne sauraient être articulés mais seulement apposés, obéissant à cet "arte povera " que décrit Bailly "à la fois un pur relevé anecdotique et [...] ce qui établirait l'écho d'une expérience, l'écho qu'il y a eu, en effet, expérience, passage dans l'obscur » $(2015: 25)$. Trace d'un passage, non pas seulement en Provence, mais dans le labyrinthe secret d'une Provence livresque, intime, lumineuse et douloureuse à la fois, Caesar's Vast Ghost renonce à l'art du récit pour nous ouvrir, d'une anecdote à l'autre, un monde qui se raconte " comme ça, en tirant sur ces petites cordes [...] un réseau de choses rapprochées » (Bailly, 2015 : 26). L'histoire de Caesar's Vast Ghost se livre dans le dépouillement de la voix de l'auteur qui nous invite à prêter attention aux vignettes arrachées à toute logique temporelle et énonciative.

21 C'est en imposant au lecteur l'ascétisme d'une prose qui refuse le récit que Durrell nous tourne une nouvelle fois vers l'Inde : celle atemporelle et symbolique d'une écriture qui se retranche dans la poésie. Chacun des vingt poèmes insérés au fil des chapitres fonctionne en effet comme " une vignette, mais sans liens, un copeau [...] un pur éclat » (Bailly, 2015 : 26). En marge de la prose, le poème est cet intrus qui vient éclairer la Provence durrellienne sans obéir à la logique narrative, sans s'inscrire dans une séquence temporelle: il ouvre un au-delà du texte qui semble flotter au-dessus des images textuelles ou photographiques, comme libéré de tout ancrage matériel. L'aporie de la prose aboutit ainsi au dire poétique.

Ouvrant et concluant l'ouvrage, les deux poèmes « Constrained by history » et « Le cercle refermé " annoncent, d'une extrémité à l'autre, une œuvre qui réside moins dans l'organisation de son dire que dans le choix et l'agencement de ses unités: mots des autres, réels ou fictifs, mots du poète, fragments d'une énonciation qui déchire le discours. Ces deux poèmes qui glissent lentement vers la première personne semblent suggérer une écriture autobiographique qui est finalement reniée. Car si l'on peut aisément entendre la voix du poète pressentant sa fin prochaine ( For the circle of the old is closing in... [...] / He'll become at last one of the Heavenly Ones », Durrell, 1990 : XIII-XIV ; « Mere time is winding down at last », Durrell, $1990: \mathrm{n}$. p.), on ne saurait ignorer, par-delà les simples échos biographiques, les liens qui se tissent d'un poème à l'autre et qui composent cette immense fresque de la mort que constitue Caesar's Vast Ghost. L'Inde surgit dès le premier poème comme l'origine de la vision intérieure du troisième œil ( the pineal eye ») qui éclaire la vie et l'art et qui entre en résonance avec la Grèce antique : "By the belly-button of Tiresias he will swear, / Or the pineal eye, the pine-cone which made him first aware / Of everything outside the bazaars of the mind " (Durrell, 1990 : XIII). Et c'est aussi vers l'Inde que nous oriente le dernier poème s'ouvrant au bord du Gange sur l'image des bûchers funéraires de Bénarès par lesquels s'accomplit le dernier passage : "The naphtha flares on river craft / Corpses floating skyward » (Durrell, 1990 : n. p.). Considérée dans le contexte de la religion hindoue la mort acquiert une toute autre signification puisque c'est en mourant à Bénarès, en y étant brûlé et en ayant ses cendres déversées dans le Gange que, selon la coutume, le croyant peut échapper au cycle des réincarnations et accéder au paradis. La mort ne se conçoit alors non pas comme une fin, mais bien comme un passage, passage symbolique et textuel qui nous conduit du début à la fin de l'œuvre : la salve du crépuscule ("Boom of the sunset gun », Durrell, 1990 : n. p.) et le sanglot solitaire du clairon ("single sobbing bugle », Durrell, $1990:$ n. p.) répondent au silence des grands mystères qui imprègne le premier poème, la fille aux neuf ventres (Durrell, 1990 : n. p.), incarnation de Mnemosyne, mère des neuf Muses et emblème de l'œuvre aux neuf 
chapitres, répond à Shiva ("the pineal eye », Durrell, 1990: XIII), la déesse douée du troisième œil qui surgit dans le premier poème dont le vers final «In you awake tonight, my love, awake like me " (Durrell, 1990: xIV) trouve son accomplissement dans le dernier poème : «So dying you begin to sleepwalk and regain your youth » (Durrell, $1990:$ n. p.).

$\mathrm{Au}$ fil des poèmes Caesar's Vast Ghost se lit comme une lente traversée vers la mort, une mort qui est à la fois décomposition du texte, des voix et des corps, mais qui est aussi éveil, surgissement d'une nouvelle forme de conscience que laisse entrevoir, au centre de l'œuvre, le poème "Béziers »: "But once the circle of initiation closes / A death-begotten insight rules » (Durrell, 1990 : 142). « Le cercle des anciens » qui se referme dans le premier poème devient le cercle initiatique qui n'est pas sans rappeler la corolle que forment sur l'eau Bruce, Piers et Sylvie après leur initiation mystique dans le désert au début du Quintette d'Avignon. Le cercle dit ainsi à la fois la fin de la vie qui se replie sur elle-même, l'enchaînement des fins et des commencements qui emprisonne l'âme et le surgissement de la lumière intérieure, l'espace à la fois vide et dense depuis lequel le poète se détache de toute forme de "vie posthume ${ }^{8}$ " pour rejoindre tous ceux "Already embalmed and sailing away ${ }^{9} »$ (Durrell, 1990 : 143).

Initiation à la mort, une mort non pas « morbide » mais sereine et lumineuse, la poésie de Caesar's Vast Ghost se détache de la nostalgie qui habite la prose et use des motifs provençaux non pas pour s'enfermer dans le topos mais pour suggérer le dernier passage. Ainsi, le poème « Why wait? » qui clôt le chapitre IV sur la tauromachie fait des taureaux camarguais des animaux chtoniens nous ouvrant des portes insoupçonnées : «Primaeval Camargue horses under sail / [...] / Curve under Roman monuments, vibrate» (Durrell, 1990 : 59). Les verbes («curve», "vibrate» sont tous deux placés sous l'accent tonique, esquissant un rythme trochaïque) qui ouvrent et terminent ce troisième vers suggèrent l'élan d'une vie nouvelle qui s'immisce dans l'interstice des ruines, qui métamorphose le temps pour en faire cette "pelote dense de durée » (" one pellet of ample duration»), un concentré d'existence qu'embrasse le souffle du yoga (" 'In yoga harness a whole reality with one soft sigh'») et au cœur duquel se rejoignent la fulgurance de l'amour ("Accelerate loving ») et le libre abandon de la mort: "The first step towards creation is to lose/Complete confidence and sort of die» (Durrell, 1990: 59). Renonçant à toute certitude, le poète convoque à la fois une temporalité et un règne nouveau : "Somehow copy the sweet conduct of these / Young olives in the spring mistral a-quiver / Silverside up [...] » (Durrell, 1990:60). Le poème est une invitation à entrer en communion avec la nature, à adopter la posture aérienne et détachée de ces « jeunes olives » qui se balancent au vent et qui ressurgissent quelques vers plus loin dans la description du visage de la belle Romaine «Delicate as young olives» (Durrell, 1990: 60). L'écriture poétique de Caesar's Vast Ghost devient ainsi l'espace privilégié de la quête du "fulcrum of repose at the heart of reality» (Durrell, 2015 [1981] : 4), ce point pivot, cette charnière depuis laquelle la méditation sur la mort bascule et nous ouvre le passage de la nostalgie (« One becomes sorry to become so soon / Just luggage left like lumber », Durrell, $1990: 60$ ) à l'espoir à travers une poésie qui se détache de la mélancolie d'un monde antique perdu pour se faire «A vessel in full sail / with a weird mystical rig » (Durrell, 1990 : 60).

Vaisseau qui lève l'ancre, équipé d'un étrange mysticisme, Caesar's Vast Ghost nous offre dans sa poésie une méditation orientale sur la mort qui n'ignore ni le douloureux attachement au passé, ni l'angoisse du dernier départ. CEuvre en partie abandonnée à la main des autres, que ce soit Mary Byrne ${ }^{10}$ qui contribua à la version finale du texte ou les poètes, écrivains, historiens, êtres réels ou imaginaires qui habitent la prose, cet ultime 
texte de Lawrence Durrell problématise de façon singulière le rapport à la mort d'un auteur qui, conscient d'avoir atteint la fin de sa vie, choisit une dernière fois la dérive des mots et des sens.

Le poème est, dans le langage, à travers lui, l'écoute de l'inconnu qui vient, ne cesse de venir. Une expérience. Qui ne reproduit pas une expérience qui lui serait préalable mais qui dans son tissu verbal la suscite et la crée. (Ancet, $2011: 82$ )

Ces paroles de Jacques Ancet permettent de mieux comprendre le jeu subtil qui s'élabore au fil du texte entre poésie et prose et par lequel Lawrence Durrell tente non pas de se saisir d'une expérience passée mais de faire résonner «des halos irradiants [...], des concrétions d'images " (Macé, 2006: 172) qui rouvrent l'expérience du monde. C'est depuis cette patrie des mots qu'annonçait «From the Elephant's Back», des mots qui racontent mais aussi des mots qui égarent comme dans ce dernier texte où l'écrivain se retire de la prose, que Lawrence Durrell invite son lecteur à se détacher des lieux communs sur la Provence pour suivre le poète sur le chemin de l'incertitude. Cette démarche, qui va du connu à l'incertain, mêlant prose et poésie, rejoint le tâtonnement propre à l'essai. Le discours sur la Provence se vide et se déplace, engageant le lecteur à rechercher dans les poèmes les dernières traces du passage de la main de l'auteur.

Les deux premières photos de Harry Peccinotti choisies par Durrell pour la première édition de Caesar's Vast Ghost soulignent cette tension aux fondements du texte. La première photo représente la maison de l'auteur à Sommières et semble offrir un ancrage biographique au poème concluant l'introduction, «Route Saussine 15 ». La deuxième, placée en regard du second poème, "Somewhere near St-Rémy ", représente un olivier près de Sommières. La torsion de l'arbre, l'ombre qu'il projette derrière lui, l'intensité de son feuillage vert-argent qui se détache sur le bleu profond du ciel rendent presque palpable cette Provence de l'âme que l'œuvre tente d'atteindre et à laquelle elle renonce dans un même geste. A l'opposé de la grande maison aux volets fermés qui semble annoncer un texte tombeau, l'olivier, affranchi de tout ancrage spatio-temporel, évoque cette terre frugale et mystique qui s'ouvre au poète mais que ni l'image ni le texte ne sauraient dévoiler. C'est dans cette dichotomie entre enfermement et ouverture, entre révélation et occultation, entre ancrage douloureux dans une mémoire au désespoir (" Despair camps everywhere » dit " Route Saussine 15 », Durrell, $1990: 8$ ) et quête d'un ultime passage, que se comprennent à la fois ce dernier texte et l'ensemble de l'œuvre de Lawrence Durrell. Cette " déchirure », qu'analyse Catherine Delmas, "qui est comme une plaie intérieure, une 'veine' ouverte par la mémoire et qui place le poète face à lui-même, à ses doubles et à son écriture » (2013:216) traverse toute l'œuvre. Le vers final du "Cercle refermé », "A disenfranchised last goodbye, / Goodbye " (Durrell, 1990: n. p.), se brise pour exprimer un adieu poignant à la vie et à l'écriture, un adieu dont la seule répétition dit la profonde mélancolie, comme si le poète échouait en fin de compte à se libérer du cycle des désirs. Ce dernier poème, qui s'ouvrait sur le passage mystique qu'accomplissent les cendres des morts sur le Gange, se clôt ainsi paradoxalement sur un adieu à tout espoir de transcendance. Il semble alors que l'écriture durrellienne, ayant cheminé pour atteindre le point d'accomplissement - «Until your pain become a literature " (Durrell, 1985 [1943, 1980] : 159) - renonce à la littérature même, rejetant dans un passé révolu le temps bienheureux où "When young and big with poems / Caressed by my heliocentric muse / With lunar leanings, I was crafty in loving" (Durrell, 1990 : n. p.). Ayant renoncé à l'époque où il était habité par sa Muse solaire, Lawrence Durrell nous plonge dans l'obscurité d'une fin au cœur de laquelle il cherche à nous laisser un signe de son passage, comme pour nous inviter à poursuivre la route : 
Cold will be the wind now, dark the storms, Ending of a visionary delight. Why to care? His art would marry the image it deserved, His sculptor's hand breaking the soft clay of old desires; mind you, the very same hand That broke the dark bread to model hunger, A presage of the faultless child in him. (Durrell, 1985 [1980] : 337) imité, Lifelines, paru en 1974. Ultime passage vers le royaume des ombres, "Certain Landfalls » annonce la chute finale dans les ténèbres, la disparition de ce "world of little mirrors in the light » (Durrell, 1985 [1980] : 158) qui ouvrait «Cities, Plains and People » et, simultanément, la résilience de l'art. La main du sculpteur qui brise le pain noir pour modeler la faim, assure ainsi la pérennité de la quête, la constance du désir. Dans le geste qui modèle " l'argile douce / des désirs anciens ", dans le « présage de l'enfant innocent » qui survit à la mort du poète, le poème nous invite à prêter attention non pas tant au chemin parcouru qu'à celui que l'œuvre littéraire poursuit en nous. Artisan des traces de son propre passage, le poète se fait ainsi passeur.

Entre présence et disparition, entre effacement et ancrage, l'écriture durrellienne nous ouvre le paradoxe silencieux qui est l'essence même de la poésie : "Music that stains / The silence remains / O echo is everywhere, the unbeckonable bird! (Durrell, 1985 [1980] : 119).

\section{BIBLIOGRAPHIE}

AleXANDRE-GARner Corinne (1996), «Regard d'exil - Naître de l'Inde : Lawrence Durrell », Cahiers du SAHIB (4), 11-25, Rennes, France : Presses universitaires de Rennes.

AlEXANDRE-GARnER Corinne (2002), « Exiled from Exile », Deus Loci, The Lawrence Durrell Journal (8), 44-57, Orlando, États-Unis : The International Lawrence Durrell Society.

AleXANDRE-GARner Corinne et Keller-Privat Isabelle (2015), « Emprunts, empreintes et disparition : l'écriture de L'Ombre infinie de César de Lawrence Durrell », Méthode! Revue de Littératures, 175-185, Bandol, France : Vallongues.

ANCET Jacques (2011), La poésie pour quoi faire ? 2/ Une enquête, 81-85, Paris, France : Presses universitaires de Paris Ouest.

BAILLY Jean-Christophe (2015), L'élargissement du poème, Paris : Christian Bourgois.

BENJAMIN Walter (1939), « Paris, capitale du XIX siècle », (« exposé » écrit directement en français par W. Benjamin, Das Passagen-Werk), en ligne sur <http://classiques.uqac.ca/classiques/ benjamin_walter/paris_capitale_19e_siecle/Benjamin_Paris_capitale.pdf> (22 janvier 2016). CooK Theodore Andrea (1905), Old Provence, Londres, Royaume-Uni : Rivingtons.

Delmas Catherine (2013), « Mémoire et cartographie de la Méditerranée dans la poésie de Lawrence Durrell », C. Reynier \& M.-E. Thérenty (dir.), Les Médiateurs de la Méditerranée, Paris, France : Geuthner, 209-223. 
DURRELL Lawrence (1985), Collected Poems 1931-1974 [1980], Londres, Royaume-Uni : Faber \& Faber. DURRELL Lawrence (1990), Caesar's Vast Ghost. Aspects of Provence, Londres, Royaume-Uni : Faber \& Faber.

DURRELl Lawrence (2008), Pied Piper of Lovers [1935], J. Gifford (dir.), Victoria, Canada : ELS

Editions

DuRRELl Lawrence (2015), « From the Elephant's Back », J. Gifford (dir.), From the Elephant's Back, Edmonton, Canada : The University of Alberta Press, 1-28.

Eco Umberto (2013), Confessions d'un jeune romancier, Paris, France : Grasset.

KeLleR-PRIVAt Isabelle (2015), « Durrell, L'Ombre infinie de César, Regards sur la Provence », Inspirations méditerranéennes, Neuilly, France : Atlande.

MACÉ Marielle (2006), Le temps de l'essai. Histoire d'un genre en France au $\mathrm{XX}^{e}$ siècle, Paris, France : Belin.

\section{ANNEXES}

Isabelle Keller-Privat est membre du CAS (Cultures Anglo-Saxonnes) et maitre de conférences - HDR au département d'anglais de l'université Toulouse 2 Jean Jaurès où elle enseigne la littérature britannique et la traduction. Lauréate de The International Lawrence Durrell Prize for New Scholarship en 2000, elle a participé à plusieurs colloques et ouvrages par des articles portant sur la conception durrellienne de l'espace-temps, l'intertexte bouddhiste, les rapports entre écriture, peinture et poésie. Elle s'intéresse également à l'œuvre de V. S. Naipaul et de J. McGregor. Elle vient de publier le premier essai critique sur les recueils poétiques de Lawrence Durrell : Between the Lines. L'écriture du déchirement dans la poésie de Lawrence Durrell (Presses universitaires de Paris Ouest : 2015).

\section{NOTES}

1. Ancien nom britannique de Jalandhar, ville de l'état du Pendjab située dans le nord-ouest de l'Inde.

2. Dans son édition critique de Pied Piper of Lovers (Durrell, 2008 [1935,], IX, XIII), James Gifford attire notre attention sur l'amendement de 1968 au Commonwealth Immigrants Act de 1962 qui fit des Anglo-indiens des apatrides, leur interdisant de retourner s'installer en Angleterre sans avoir préalablement obtenu un visa.

3. Les premiers recueils de poèmes, Quaint Fragments, Ten Poems et Transition: Poems, paraissent successivement en 1931, 1932 et 1935. Le roman Panic Spring suit la publication de Pied Piper of Lovers en 1937 et The Black Book parait en 1938.

4. La majorité des exemplaires du premier tirage fut détruite pendant le Blitz et le petit nombre qui resta en circulation en fit un texte privé, connu d'un lectorat limité jusqu'à sa réédition par James Gifford en 2008.

5. Le texte français avait été publié dans la Revue parlée du Centre Georges-Pompidou de mars 1981 et n'est consultable qu'à la Bibliothèque de recherches Lawrence Durrell de l'université Paris Ouest, qui détient également l'une des versions anglaises du texte (L. Durrell, «From the Elephant's Back, text amended and slightly expanded of a lecture first given in French at the Centre Pompidou, Paris, April 1, $1981 »$, p. 1. Ref $n^{\circ}: 2199 /$ Réserve). Les traductions 
successives ont abouti à des remaniements et à des développements du propos de l'auteur, ce qui explique que nous ayons choisi de le citer ici en anglais dans la version définitive retenue par James Gifford.

6. Un exemplaire de cet ouvrage est consultable à la Bibliothèque de recherches Lawrence Durrell de l'université Paris Ouest.

7. Il suffit de penser en particulier aux propos antisémites d'Aldo ou de Cunégonde (voir Alexandre-Garner et Keller-Privat, 2015).

8. Remarquer l'orthographe inhabituelle de l'adjectif anglais «post-humous» qui souligne l'arrachement du poète à la terre (Durrell, $1990: 143$ ).

9. Ce vers de « Béziers » annonce et prépare « corpses floating skywards » dans le dernier poème où le lent glissement des corps sur l'eau oriente le regard vers l'horizon, préparant le passage de l'horizontalité à la verticalité qui s'accomplit dans le rêve à demi éveillé du vers "you begin to sleepwalk and regain your youth " (Durrell, 1990 : n. p.).

10. Durrell, dont la santé ne cessait de se dégrader, et dont le premier manuscrit avait été refusé par son éditeur Faber en 1986, eut recours à l'aide de Mary Byrne, pour remanier la composition du volume. Mary Byrne avait soutenu une thèse en 1980 sur Le Quatuor d'Alexandrie avant de rencontrer Lawrence Durrell ; par la suite, elle enseigna à l'université de Caen et exerce à présent comme traductrice.

\section{RÉSUMÉS}

Cet article se propose d'étudier le cheminement de l'écriture de Lawrence Durrell à partir du premier récit autobiographique, peu connu, Pied Piper of Lovers (1935), afin de comprendre comment l'expérience de l'Inde natale permet à l'auteur de forger un rapport particulier à l'espace et à l'écriture. Ses publications ultérieures, en particulier sa conférence de 1981 « From the Elephant's Back » et son essai sur la Provence Caesar's Vast Ghost (1990), ne cessent en effet de revisiter l'Inde, non plus cette fois comme le lieu d'un ancrage autobiographique mais comme un lieu symbolique d'accès au monde et à la littérature. Le dernier texte de Lawrence Durrell s'éclaire ainsi dès lors que le lecteur saisit, derrière les contours de la Provence, l'ombre de l'Inde qui informe la décomposition de l'espace provençal au profit d'une écriture qui se retranche dans la poésie, dernier exercice d'ascèse littéraire et philosophique d'un écrivain qui se retire du monde.

This paper wishes to explore the maturing process of Lawrence Durrell's art starting from his first and lesser known autobiographical narrative, Pied Piper of Lovers (1935), in order to understand how his experience of his native land, India, enabled him to carve out a specific relationship to space and writing. India never ceases to insist throughout his later publicationsnamely his 1981 conference "From the Elephant's Back" and his essay on Provence, Caesar's Vast Ghost (1990)-but the autobiographical attachment progressively fades away as the land of birth is recast into a symbolic space wherein the writer is reborn to the world and to literature. Durrell's last opus reveals its hidden meaning to the reader as soon as the latter perceives the shadow of India which underlies the Provencal space and paves the way for its decomposition as the writer withdraws into the poems, his last exercise in literary and philosophic asceticism before withdrawing from life. 
INDEX

Keywords : Lawrence Durrell, India, Provence, space and writing, autobiography, poetry, philosophy, essay

Mots-clés : Lawrence Durrell, Inde, Provence, espace et écriture, autobiographie, poésie, philosophie, essai

\section{AUTEUR}

\section{ISABELLE KELLER-PRIVAT}

Maître de conférences - HDR à l'université Toulouse 2 Jean Jaurès (CAS, Cultures Anglo-Saxonnes) 